\title{
ASSESSING THE DYNAMIC BEHAVIOR OF WSN MOTES AND RFID SEMI-PASSIVE TAGS FOR TEMPERATURE MONITORING
}

\author{
Ricardo Badia-Melis ${ }^{1 *}$, Javier Garcia-Hierro², Luis Ruiz-Garcia ${ }^{1}$, Tatiana Jiménez- \\ Ariza $^{1}$, José Ignacio Robla Villalba ${ }^{2}$, Pilar Barreiro ${ }^{1}$ \\ ${ }^{1}$ Laboratorio de Propiedades Físicas Y tecnologías Avanzadas en Agroalimentación, Universidad \\ Politécnica de Madrid, CEI-Campus Moncloa. ETSI Agrónomos, Edificio Motores, Avda. \\ Complutense s/n, 28040 Madrid, Spain \\ ${ }^{2}$ Sensors Technology Laboratory, Centro Nacional de Investigaciones Metalurgicas, Consejo Superior \\ de Investigaciones Científicas (CENIM-CSIC), Avenida Gregorio del Amo, 8. 28040, Madrid Spain \\ ${ }^{*}$ Corresponding author. E-mail: ricardobadia@live.com
}

\begin{abstract}
A notorious advantage of wireless transmission is a significant reduction and simplification in wiring and harness. There are a lot of applications of wireless systems, but in many occasions sensor nodes require a specific housing to protect the electronics from hush environmental conditions. Nowadays the information is scarce and nonspecific on the dynamic behaviour of WSN and RFID. Therefore the purpose of this study is to evaluate the dynamic behaviour of the sensors. A series of trials were designed and performed covering temperature steps between cold room $\left(5^{\circ} \mathrm{C}\right)$, room temperature $\left(23^{\circ} \mathrm{C}\right)$ and heated environment $\left(35^{\circ} \mathrm{C}\right)$. As sensor nodes: three Crossbow motes, a surface mounted Nlaza module (with sensor Sensirion located on the motherboard), an aerial mounted Nlaza where the Sensirion sensor stayed at the end of a cable), and four tags RFID Turbo Tag (T700 model with and without housing), and 702-B (with and without housing). To assess the dynamic behaviour a first order response approach is used and fitted with dedicated optimization tools programmed in Matlab that allow extracting the time response $(\tau)$ and corresponding determination coefficient $\left(r^{2}\right)$ with regard to experimental data. The shorter response time $(20.9 \mathrm{~s})$ is found for the uncoated $\mathrm{T} 700$ tag which encapsulated version provides a significantly higher response (107.2 s). The highest $\tau$ corresponds to the Crossbow modules (144.4 s), followed by the surface mounted Nlaza module (288.1 s), while the module with aerial mounted sensor gives a response certainly close above to the T700 without coating (42.8 s). As a conclusion, the dynamic response of temperature sensors within wireless and RFID nodes is dramatically influenced by the way they are housed (to protect them from the environment) as well as by the heat released by the node electronics itself; its characterization is basic to allow monitoring of high rate temperature changes and to certify the cold chain. Besides the time to rise and to recover is significantly different being mostly higher for the latter than for the former.
\end{abstract}

\section{Keywords}

Metrology, smart sensor, monitor, logistics, sensor network

\section{Introduction}

WSN and RFID are changing and entering in a new phase and is said to improve the performance of agricultural world. A notorious advantage of wireless transmission is a significant reduction and simplification in wiring and harness. Also, wireless technologies show several differences and advantages over previous technologies like barcode. RFID tags do not require direct line of sight to the reader and thus can be embedded in a item, placed inside the packing or injected inside the body of animals. RFID can be read even more than $100 \mathrm{~m}$ of distance, and due to it reasonable price and the high read rates you can also use as much as units as you need, for instance more than 100 tags per second (RuizGarcia et al., 2011). Moreover RFID tags have enough memory to store data (up to 4kB in 
passive, $1 \mathrm{MB}$ in active tags) such as temperature and humidity; and the WSN often uses external memories or computer`s hard disk.

There are a lot of applications of wireless systems such as: traceability by means of RFID, food packaging, history checking and contamination, or inventory control and food inspection (Wang et al., 2006).

Relevant environments for the application of wireless systems include monitoring of fruit and vegetable containers and cold-storage facilities, or monitoring quality and senescence of specialty crops during transport (Ruiz-Garcia et al., 2009). The economic impact of product losses stays around 10\% in Europe (6-7\% in retailers) and 15\% in USA (Pang et al., 2012), while reaching $30 \%$ in developing countries (mostly to the lack of temperature control). In either of the cases, with transport distances above several thousand of kilometers (Jedermann et al., 2011).

Pang et al. (2011) explicitly declare that Food chains has become highly distributed, heterogeneous, cooperative, and globalized with extremely diverse requirements, and thus declares the need for convergence of all the necessary technical requirements (with dedicated analysis for wireless technologies), corresponding operation models, networking protocols, hardware (WSN versus RFID), and software. According to the authors, a scenario is an abstraction of a class of deployment environments, and so a particular scenario may appear multiple times at different positions among the entire sequence link, from produce and manufacturers to sell or consumers. Besides, depending on the scenario there is a specific need for sensors: store scenario requires gas concentration monitoring (oxygen, ethylene or carbon dioxide), while transport demands GPS and acceleration measurements, while the variety of scenarios also provides specific facilities like WIFI (store and sell scenario), or accessible power supply (sell and consumer scenario).

Jedermann et al., 2011 focus mainly in the use of WSN in transport, and more precisely in the high attenuation of signal in packed food which has turned out to be the major problem for monitoring packed food transports. According to the authors, about $20 \%$ of the sensor data are lost because there is no physical route possible between a sensor node and the base station, while additional sensor data are lost because the protocols are not able to detect the correct routing ( $4 \%$ of the sensor data). Another conclusion of this study is the fact that electronics has to be protected against high humidity, condensed water, and mechanical stress during transshipments, while automated localization of the sensors inside the container could be very useful, because it cannot be guaranteed that the workers in the packing station may report the sensor positions properly.

In many occasions sensors nodes require a specific housing to protect the electronics from hush environmental conditions, since this kind of equipment was not originally designed for them, i.e. meteorological data acquisition system (Lee et al., 2010), however this effort is always shows as contra wise effect the lack of sensitivity of sensors, in a Penelope-like strategy.

Interestingly, up to date there is no scientific information available on the dynamic behavior of WSN and RFID as related to sensor housing or mounting on the base electronics. The aim to this study is therefore to assess the dynamic behavior of the sensors as crucial for a proper characterization of history reconstruction.

\section{Material and Methods}

A series of trials were designed and performed with some of the technologies that could represent the future in monitoring processes, processes as mentioned in the introduction of the paper. This devices covering temperature steps between cold room $\left(5^{\circ} \mathrm{C}\right)$, room temperature $\left(23^{\circ} \mathrm{C}\right)$ and heated environment ( stove at $35^{\circ} \mathrm{C}$ ). 
This test has included wireless modules (with ZigBee protocol) type Crossbow Iris (IRIS_XM2110) with sensor card which includes sensors of light, acceleration, temperature, humidity and pressure (MTS card 420), a standard Nlaza module (with sensor Sensirion located on the motherboard), and a Nlaza module modified (Sensirion sensor stayed at the end of a cable) and finally four RFID cards has been used, two 702-B Turbo Tag (one with coating and one without coating), two Turbo Tag 700 (with coating and without this coating).

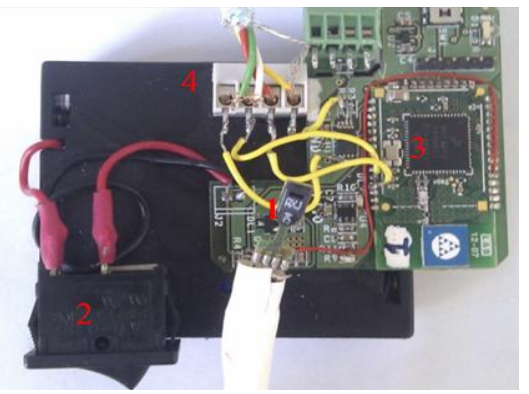

FIGURE 1: Nlaza module with the Sensirion sensor at the end of a cable

The modified module is provided with: (1) Temperature and relative humidity sensor. Sensirion SHT 15, (2) Switch on/off, (3) Transmitter, receptor and (4) Electrical connector.

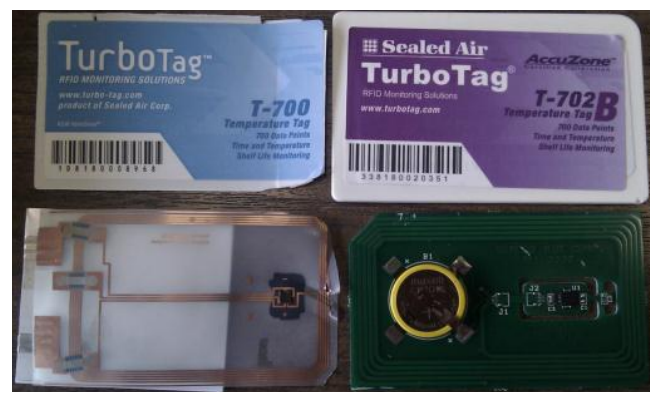

FIGURE 2: Turbo Tags T-700 and T702 with and without coating

After several unsuccessful attempts, it was possible to carry out the experiment detailed below, were RFID cards and Crossbow nodes were used. Nlaza modules were also used in a later experiment under the same set-up: shifting from $5^{\circ} \mathrm{C}$ (refrigerator) to $35^{\circ} \mathrm{C}$ (controlled temperature oven).

Is necessary to keep the sensors far away as much as possible of the motherboards, battery and battery case and unnecessary coatings, and therefore a Turbo tag of each type has been opened, and is left exposed the sensor to air, thus it can be seen the thermal inertia effect of the coatings

We started the test by placing three Crossbow modules, one in the stove (at $35^{\circ} \mathrm{C}$ ), other one in the fridge (with a temperature of $5^{\circ} \mathrm{C}$.) and the last into a room temperature, because was needed to have reference temperatures to compare On the other hand were positioned in the fridge the one Crossbow module and RFID tags. At that time the Crossbow modules were activated and the computer started to register the data (every 3 seconds).

After 20 minutes it was estimated that had stabilized at the temperature of the refrigerator, then RFID tags were programmed every 4 seconds (minimum interval that leaves the manufacturer's software), started the experiment, at 8:02 am April 26, 2011.

The module and the Turbo Tags were changed to the stove at 8:15 am, these remained 20 minutes inside the stove, enough time to observe temperature stabilization between the reference module inside the stove and the module just insert (is possible see the table temperatures in real time showing the software of the Crossbow modules). 
After that were transported to the refrigerator, at $8: 35 \mathrm{am}$, there remain 30 minutes, again until to see stabilization.

For the last time to the stove, at 9:06 am the process was repeated, to facilitate data analysis if the first change had errors and improve the quality of information.

\subsection{Procedure of the data analysis.}

For data analysis, dedicated routines have been developed in Matlab for an accurate characterization of the time response of the sensors, based on the equation that represents the behavior of a first order system (Pallas, 1998), that is to say there is an element that absorbs and relishes energy: the sensor house. Therefore, the relationship between the input $x(t)$ and the output $y(t)$ is made by a differential equation type (Eq. 1)

$$
a 1 * \frac{d y(t)}{d t}+a 0 * y(t)=x(t)
$$

The corresponding transfer function is shown in Equation 2.

$$
\frac{Y(s)}{K(s)}=\frac{k}{\tau s+1}
$$

Where $k=1 / a_{0}$ is called static sensitivity, and $T=a_{1} / a_{0}$ is known as the system time constant. The natural frequency $\omega c$ is given by $1 / \mathrm{T}$. The system is characterized therefore by two parameters $\mathrm{k}$ for the static response and $\omega \mathrm{c}$ or $\tau$ for the dynamic response.

The response of a first order system when using a step-shaped temperature decrease also follows an exponential downward trend (Equation 3).

$$
y=k * x * e^{-t / \tau}
$$

While the upward trajectory under step-shape excitation is expressed by Equation 4 .

$$
y=k * x *\left(1-e^{-t / \tau}\right)
$$

Considering that in our test we shift from a base temperature $\left(5^{\circ} \mathrm{C}\right)$ to an upper bound limit $\left(35^{\circ} \mathrm{C}\right)$ and back to the initial temperature $\left(5^{\circ} \mathrm{C}\right)$, previous mathematical expressions are adapted to Equations 5 and 6.

$$
\begin{gathered}
y=a *\left(1-e^{-x / b}+c\right) \\
y=a *\left(e^{-x / b}+c\right)
\end{gathered}
$$

Being: "a" the temperature step (30 ำ temperature shift), " $\mathrm{x}$ " the time interval, "b" standing for " $\tau$ " which is the sensor response time (s), and "c" being the initial annealing temperature for the upward trajectory and the final stabilization in the downward trend.

\section{Results}

A comparison is made on the average reaction rate of the sensors (Turbo Tag or Sensirion) when shifting between 5 and $35{ }^{\circ} \mathrm{C}$, with the result of a faster response corresponding to T700 (without coating) which surpasses $0.18^{\circ} \mathrm{C} / \mathrm{s}$, followed by the aerial-mounted Nlaza module, and the T702 card without coating, this fact highlights the influence of coating and sensor montage on the motherboards with regard to the reaction rates. Besides, encapsulated RFID tags show to be faster than the Crossbow module, while the lowest speed $\left(0.04^{\circ} \mathrm{C} / \mathrm{s}\right)$ corresponds to surface -mounted Nlaza module, where the sensor is soldered onto the motherboard. Reaction times are plotted in figure 3 , while first order response parameters are provided in Table 1. 


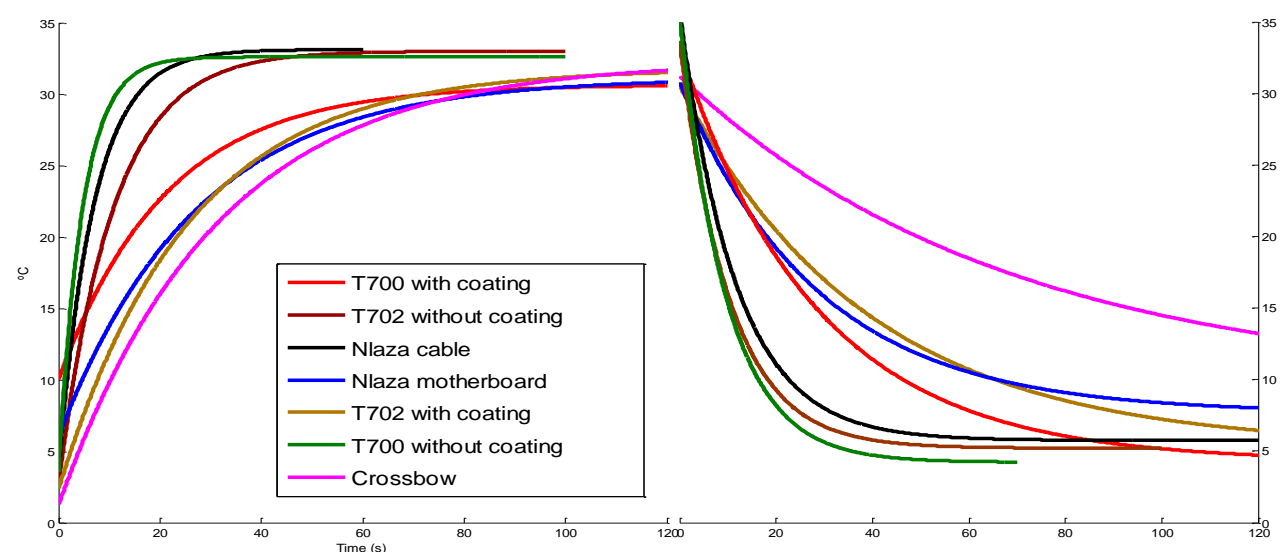

FIGURE 3: Fitted shapes of the sensor's response times

Figure 3 shows the fits of the temperature rise and fall by the change from the fridge to the stove and vice versa. The most important thing of this graphic is to look at the different slopes. It shows a lower rate reaction tags without coating and Nlaza modified module, with biggest slopes. After that goes the T700 tag and T702 with coating, the Crossbow module later and finally the Nlaza module with the softer slope. In the downward shapes can be seen that the tags without coating together with the modified Nlaza have again the shorter response, but now the bigger response belongs to the Crossbow module.

The script created in Matlab gives the response times and the fits with $r^{2}$ that is showed in the table below. At the same time is obtained also with this script, the graphic (Fig3).

TABLE 1: Response times and $r^{2}$

\begin{tabular}{|c|c|c|c|c|c|}
\multicolumn{2}{c}{} & \multicolumn{2}{c}{ Upward shape } & \multicolumn{2}{c|}{ Downward shape } \\
\cline { 3 - 6 } \multicolumn{1}{c|}{ Device } & Status & $\boldsymbol{\tau}(\mathbf{s})$ & $\mathbf{r}^{2}$ & $\boldsymbol{\tau}(\mathbf{s})$ & $\mathbf{r}^{2}$ \\
\hline \multirow{2}{*}{ T700 } & With coating & $107.2 \pm 0.3$ & 0.99 & $115.0 \pm 0.3$ & 0.99 \\
\cline { 2 - 6 } & $\begin{array}{c}\text { Without } \\
\text { coating }\end{array}$ & $20.9 \pm 0.3$ & 0.99 & $45.8 \pm 0.6$ & 0.98 \\
\hline \multirow{2}{*}{ T702 B } & With coating & $131.2 \pm 0.3$ & 0.99 & $156.8 \pm 0.3$ & 0.99 \\
\cline { 3 - 6 } & $\begin{array}{c}\text { Without } \\
\text { coating }\end{array}$ & $53.8 \pm 0.6$ & 0.98 & $57.6 \pm 0.9$ & 0.97 \\
\hline Nlaza & Motherboard & $126.4 \pm 0.3$ & 0.99 & $184.0 \pm 0.3$ & 0.99 \\
\cline { 2 - 6 } & air & $42.8 \pm 0.3$ & 0.99 & $58.4 \pm 0.3$ & 0.99 \\
\hline $\begin{array}{c}\text { Crossbow Iris } \\
\text { MTS 400 }\end{array}$ & Motherboard & $144.4 \pm 1.2$ & 0.96 & $254.0 \pm 0.6$ & 0.98 \\
\hline
\end{tabular}

Table 1, shows the settings of the measured temperatures, the validity of the adjustments is corroborated by the values of $r^{2}$, all very close to unit. On the other hand, the $\tau$ obtained show that response times are lower in the unencapsulated T700 tag than the encapsulated, with a rise of temperatures in 20.9 seconds $\left(\tau=20.9\right.$ seconds and $\left.r^{2}=0.99\right)$, while the encapsulated version provides $\tau=107.2$ seconds and $r^{2}=0.99$. Followed by the module with aerial-mounted Nlaza with $\tau=42.8$ seconds $\left(r^{2}=0.99\right)$, and followed by the T702 (53.8 s time response).

These response time (required to reach $63 \%$ of static response) contrasting with the highest $\tau$ which corresponds to the Crossbow module with $\tau=144.4$ seconds $\left(r^{2}=0.96\right)$, followed by the surface-mounted Nlaza module $\left(\tau=126.4\right.$ seconds $\left.r^{2}=0.99\right)$.

In the downward trend, the T700 tag shows a response time of 45.8 seconds, followed by the T702 with 57.6 seconds and the aerial-mounted Nlaza module in the third position (58.4 
seconds). The largest response time corresponds to the Crossbow module (254 seconds), while the Nlaza module that has Sensirion welded to the motherboard reports a response time of 184 seconds.

\section{Conclusions}

The dynamic response of temperature sensors within wireless and RFID nodes is dramatically influenced by the way they are housed (to protect them from the environment) as well as by the heat released by the node electronics itself; its characterization is basic to allow monitoring of high rate temperature changes and to certify the cold chain.

In this study the fastest response time stays around 20s which indicates that temperature cycles of higher frequency cannot be addressed with available wireless technologies. On the other end, the slowest response (144 s) corresponds to surface-mounted sensors which refers the need of a dedicated design.

\section{Acknowledgements}

The funding of this work has been covered by the MICINN with the project "Sensórica inteligente para el Control de la Calidad en línea de procesos alimentarios Smart-QC" (ALG2008-05267-C03-03), by MITYC, plan Avanza with the project "Plataforma de Control Logístico Avanzado para el seguimiento de la cadena de frío de productos Sanitarios y Agroalimentarios CLARISA" (TSI-020100-2009-851),

And by the European Commission with the project InsideFood "Integrated, sensing and imaging devices for designing, monitoring and controlling microstructure of foods" (FP7KBBE-2B-226783)

\section{References}

Jederman, R., Becker, M., Görg, C., Lang, W., 2011. Testing network protocols and signal attenuation in packed food transports. International Journal of Sensor Networks. Volume 9, Number 3-4/2011.170-181.

Ning Wang, Naiqian Zhang, Maohua wang., 2006. Wireless sensors in agriculture and food industry-Recent development and future perspective. Computers and electronics in Agriculture50(2006)1-14

Pallás Areny, R. (1998). Sistemas de primer orden Sensores y acondicionadores de señal 3ed S. A. MACOMBO.

Pang, Z., Chen, Q., \& Zheng, L. (2012). Scenario-based design of wireless sensor system for food chain visibility and safety. In Y. Wu (Ed.), Advances in computer, communication, control and automation (pp. 541-548) Springer Berlin Heidelberg.

Ruiz-Garcia, L., Lunadei, L., 2011. The role of RFID in agriculture: Applications, limitations and challenges. Computers and electronics in agriculture 79(2011)42-50

Ruiz-Garcia, L., Lunadei, L. Barreiro, P. Robla, J.I. 2009. A Review of Wireless Sensors technologies and Applications in agriculture and food industry: State of the art and Actual Trends. Sensors ISSN 1424-8220. Sensors, 2009, 9 (6), pp. 4728-4750.

W.S. Lee, V. Alchanatis, C. Yang, M. Hirafuji, D. Moshou, C. Li., 2010. Review. Sensing technologies for precision specialty crop production. Computers and Electronics in Agriculture 74 (2010) 2-33. 